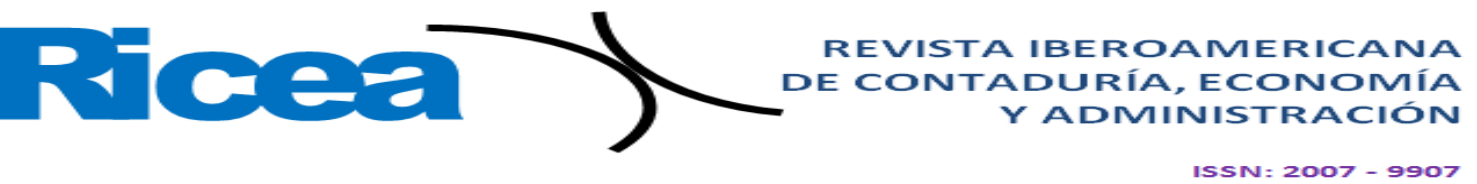

https://doi.org/10.23913/ricea.v8i16.139

Artículos Científicos

\title{
Análisis correlacional del bienestar psicológico en el trabajo, liderazgo transformacional y confianza en el líder: caso de una mype industrial
}

Correlational analysis of psychological well-being at work, transformational leadership and trust in the leader: Case of an industrial small Enterprise

Análise correlacional do bem-estar psicológico no trabalho, liderança transformacional e confiança no líder: caso de um tipo industrial

María Brenda González Herrera Universidad Juárez del Estado de Durango, México mbrendagh@gmail.com https://orcid.org/0000-0003-3697-2074

José Gerardo Ignacio Gómez Romero Universidad Juárez del Estado de Durango, México gerardoignaciog@yahoo.com.mx https://orcid.org/0000-0002-7900-9141

Ernesto Geovani Figueroa González Universidad Juárez del Estado de Durango geovani.figueroa@ujed.mx https://orcid.org/0000-0002-6322.6133 


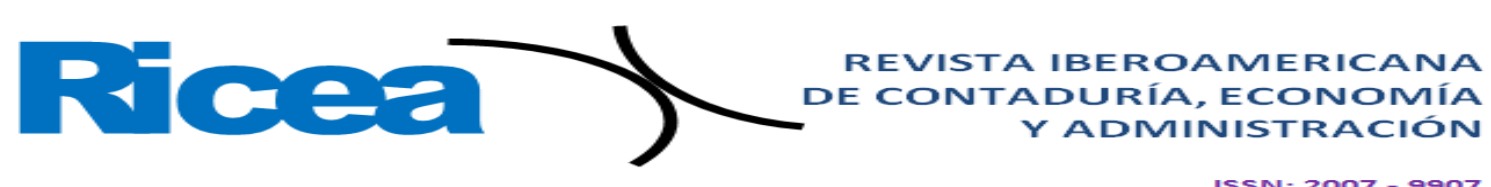

\section{Resumen}

En el presente estudio se examinó la correlación existente entre el bienestar psicológico en el trabajo, el liderazgo transformacional y la confianza en el líder según la percepción de trabajadores de una pequeña industria ubicada en la ciudad de Durango, estado de Durango, México. El objetivo de la investigación fue analizar las posibles relaciones entre las percepciones de los trabajadores sobre el estilo de liderazgo y su bienestar psicológico, así como verificar el papel de la confianza sobre tales relaciones. Se llevó a cabo un estudio de caso cuantitativo, de corte transversal correlacional. En concreto, se realizó un censo a una población de 23 trabajadores. El análisis de correlaciones mostró importantes asociaciones entre el liderazgo transformador (consideración individualizada, motivación inspiradora, influencia idealizada y estimulación intelectual), la confianza en el gerente, así como el bienestar psicológico en el trabajo. Desde una perspectiva práctica, los aportes científicos proveen una herramienta potencial para el diagnóstico del bienestar psicológico en el trabajo con un rigor metodológico en la métrica, lo cual permite comprender las nociones de bienestar y liderazgo, así como plantear estrategias organizacionales para las mypes.

Palabras clave: gestión empresarial, liderazgo, pequeñas empresas.

\section{Abstract}

The present study examined the correlation between transformational leadership, psychological well-being at work and trust in leader in the perception of workers in a small transformation industry located in the city of Durango. State of Durango, Mexico. The objective of the research was to analyze the possible relationships between workers' perceptions of leadership style and their psychological well-being, as well as to verify the role of trust in such relationships. A quantitative, cross-sectional correlational case study was carried out, carrying out a census of a population of 23 workers. The analysis of correlations shows important associations between transformative leadership (individualized consideration, inspiring motivation, idealized influence and intellectual stimulation). trust in the manager, as well as psychological well-being at work. From a practical perspective, scientific contributions provide a potential tool for the diagnosis of psychological well-being at work with a methodological rigor in the metrics, which allows understanding the notions of well-being and leadership and proposing organizational strategies for Micro and Small Enterprises (MSE’s).

\section{(c) 9 (F)}




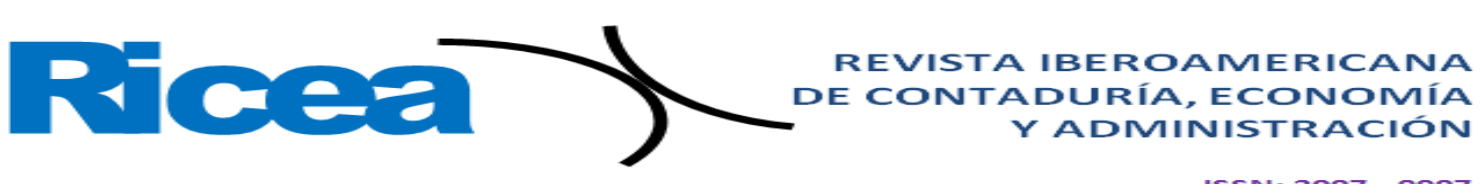

Keywords: business management, leadership, small enterprises.

\section{Resumo}

Este estudo examinou a correlação entre bem-estar psicológico no trabalho, liderança transformacional e confiança no líder de acordo com a percepção dos trabalhadores em uma pequena indústria localizada na cidade de Durango, estado de Durango, México. O objetivo da pesquisa foi analisar as possíveis relações entre as percepções dos trabalhadores sobre o estilo de liderança e seu bem-estar psicológico, bem como verificar o papel da confiança nessas relações. Foi realizado um estudo de caso quantitativo e transversal. Especificamente, foi realizado um censo em uma população de 23 trabalhadores. A análise de correlação mostrou associações importantes entre liderança transformadora (consideração individualizada, motivação inspiradora, influência idealizada e estímulo intelectual), confiança no gerente e bem-estar psicológico no trabalho. Do ponto de vista prático, as contribuições científicas fornecem uma ferramenta potencial para o diagnóstico do bem-estar psicológico no trabalho, com um rigor metodológico nas métricas, o que nos permite entender as noções de bem-estar e liderança, bem como propor estratégias organizacionais para o mipes.

Palavras-chave: gestão de negócios, liderança, pequenas empresas.

Fecha Recepción: Diciembre 2018 Fecha Aceptación: Mayo 2019

\section{Introducción}

Las micro, pequeñas y medianas empresas (mipymes) registran una tendencia de crecimiento mundial, de tal modo que en Latinoamérica y México se han convertido en los últimos decenios en un importante motor de la economía que genera de manera significativa empleo y autoempleo. De acuerdo con Sansores y Navarrete (2018), en el mundo estas constituyen más de $95 \%$ de las unidades económicas, porcentaje que en Latinoamérica aumenta hasta $99 \%$, y en ambos casos emplean a más de $60 \%$ del total de trabajadores.

Aun así, la presente investigación solo se centra en el análisis de las micro y pequeñas empresas (mypes), ya que las medianas cuentan con estructuras más definidas en sus procesos y mecanismos de funcionamiento, lo cual las acerca más a las grandes empresas

\section{(C) $98 \%$}




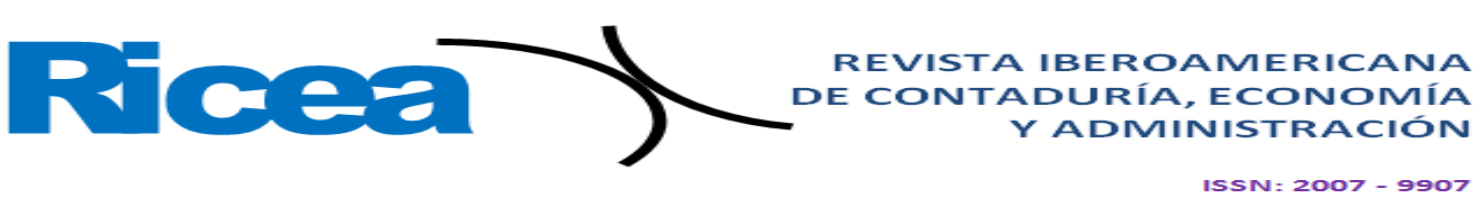

(Posada, Aguilar y Peña, 2016). En concreto, el enfoque se fija en el estudio de caso de una pequeña empresa industrial (que cuenta con 26 trabajadores, incluido el gerente) perteneciente al sector de la transformación ubicada en la ciudad de Durango, Durango, México.

Para su clasificación, se acudió al Diario Oficial de la Federación (2009) —aún vigente- - en el cual se menciona que uno de los criterios básicos para la clasificación de las mipymes es el número de sus empleados; es decir, las microempresas tienen hasta 10 trabajadores; las pequeñas, entre 11 y 50; las medianas, entre 50 y 250, y las grandes más de 251.

En México, las mypes conforman $99.8 \%$ de las empresas y $72.3 \%$ de las fuentes de empleo. Sin embargo, su productividad es relativamente baja, ya que en conjunto solo aportan $25 \%$ del producto interno bruto (PIB), según datos del Instituto Nacional de Geografía e Informática (Inegi), actualizados hasta 2018. Asimismo, y según Posada et al. (2016) — quienes también se basan en datos ofrecidos por el Inegi-, los principales problemas de las mypes son la competencia excesiva, los escasos clientes, la falta de crédito, las mínimas ganancias, los problemas con las autoridades y el pago entre proveedores y clientes, razones por las que su esperanza de vida promedio es de 7.7 años. De hecho, más de $80 \%$ de estas no sobrevive a los dos años. Esto significa que la gestión como variable tiene un rol primordial que antecede al liderazgo.

A lo largo de 30 años — como señalan Posada et al. (2016)—, se ha analizado la problemática anteriormente descrita para encontrar mecanismos que ayuden a las mypes no solo a crecer, sino también a desarrollarse y a contar con valor agregado para que en su entorno social exista una mejor redistribución de la riqueza.

En tal sentido, Avendaño y Perrotini (2015) — con base en datos del Banco de México (2013) y del Banco Mundial (2014) — explican que México ha tenido un lento crecimiento en esta área desde la década de 1980 debido a las fallas de mercado, de gobierno y de diversas variables económicas y financieras.

Estos factores limitan las posibilidades de acción de los microempresarios y pequeños empresarios en cuanto a elevar el potencial de sus recursos financieros y materiales, aunque mantienen sus esfuerzos en relación con el capital humano, factor indispensable no solo para promover la productividad, la competitividad y la rentabilidad de sus empresas, sino también para evitar que estas desaparezcan en los primeros dos años de fundación. Para fortalecer este

\section{(c) $\rightarrow$ Fै}




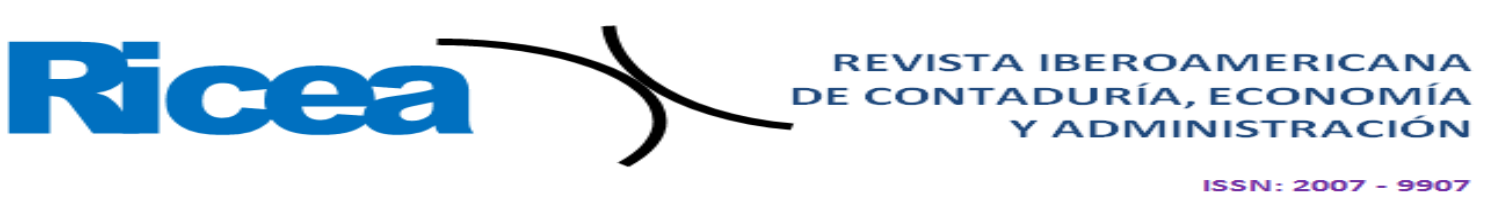

capital, por supuesto, se necesitan políticas claras en cuanto a promociones, remuneraciones, evaluaciones y capacitaciones, variables que surgen de la gestión.

Por este motivo, en el presente estudio se busca determinar los factores de valor y de ventaja competitiva que representan el capital humano desde el concepto de bienestar psicológico en el trabajo bajo el estilo de liderazgo transformacional, así como la implicación de la confianza en el líder para establecer la relación entre las variables, lo cual permitirá conocer posibles herramientas para las estrategias efectivas en la gestión del capital humano.

Una gestión efectiva del capital humano puede ser significativa para la permanencia y el éxito de las mypes. El reto, no obstante, es identificar y administrar las condiciones para que personas con diferentes necesidades, conocimientos, actitudes y aprendizajes no solo consigan factores de trabajo óptimo, sino también se sientan plenas y realizadas en su vida, lo cual puede beneficiar al negocio en cuanto a productividad, innovación y demás objetivos propuestos.

En la presente investigación se llevó a cabo un estudio de caso cuantitativo, de corte transversal correlacional, para lo cual se aplicó un censo a una población de 23 trabajadores. Las hipótesis evaluadas fueron las siguientes:

- H1. Existe una correlación entre el estilo de liderazgo transformacional y el bienestar psicológico en el trabajo.

- H2. Existe una correlación entre el liderazgo transformacional y la confianza en el líder.

- H3. Existe correlación entre la confianza en el líder y la percepción del bienestar psicológico en el trabajo.

Los resultados expresan la percepción que tienen los trabajadores y muestran un importante nivel de correlación entre el liderazgo transformacional (consideración individualizada, motivación inspiradora, influencia idealizada y estimulación intelectual), la confianza en el gerente, así como el bienestar psicológico en el trabajo.

Desde una perspectiva práctica, los aportes científicos proveen una herramienta potencial para el diagnóstico del bienestar psicológico en el trabajo con un rigor metodológico en la métrica, lo cual permite comprender las nociones de bienestar y liderazgo y plantear estrategias organizacionales para las mypes.

\section{(C) $\rightarrow \Leftrightarrow \Leftrightarrow$}




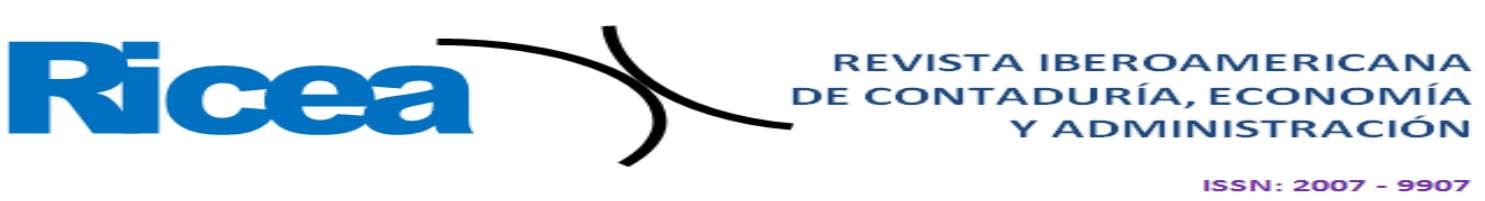

En un inicio, las prácticas dirigidas a la promoción del bienestar laboral se centraron en programas sociales tendientes a mejorar las condiciones morales y mentales del trabajador; posteriormente, en proyectos orientados a modificar las condiciones de trabajo o a ofrecimientos de prestaciones especiales que complementaran los ingresos y condiciones sociales del trabajador; después, al logro de estrategias para el incremento de la lealtad, la motivación y, por último, a la búsqueda de condiciones que mejoraran de manejar integral la calidad de vida laboral (Barley y Kunda, 1992).

En años recientes comenzaron a tomar relevancia estudios que enfatizan la relación de la felicidad en los seres humanos y el efecto que produce en el trabajo, y se determinó que es una variable que afecta a la otra de manera significativa. Posteriormente, se ha demostrado que la felicidad es solo un componente hedónico del aspecto cognitivo del ser humano, por lo que los estudios han ampliado la conceptualización y han abarcado aspectos relativos al desarrollo personal, lo que ha derivado en un enfoque eudamónico, que considera otros aspectos de la vida que por sí solos los satisfactores de placer no cumplen. Por ello, las investigaciones han procurado conceptualizar ambos enfoques con el término bienestar psicológico, y se ha determinado que cuanto mayor es este, se consigue un mejor desempeño y atención en las tareas (Diener y Biswas-Diener 2002; Diener y Seligman, 2004; Seligman, Ernst, Gillham, Reivich y Linkins, 2009).

En efecto, los individuos con niveles más elevados de bienestar evaluativo tienen más capacidad para proyectar su futuro (esta parte se relaciona con la perspectiva eudaimónica) que otros individuos con menores perspectivas de superación, los cuales se centran más en la experiencia diaria del bienestar, precisamente porque sus perspectivas de futuro son mucho menos seguras (lo que se relaciona estrechamente con la perspectiva hedónica) (Adler et al., 2013; Adler, Dolan y Kavetsos, 2014).

De acuerdo con los resultados de los trabajos citados, se han efectuado algunas aplicaciones al campo laboral, sobre todo en sociedades estadounidenses y europeas a través de la gestión de mejoras para las condiciones laborales con resultados positivos. Sin embargo, también se ha puesto en evidencia que el bienestar no constituye solo aspectos objetivos como el salario o el acceso a servicios públicos, sino también variables afectivas del ser humano, ya que se deben considerar elementos cognitivos, así como la importancia que tiene para las personas definir el sentido de la vida y los factores necesarios para su crecimiento personal.

\section{(c) 9 (9)}




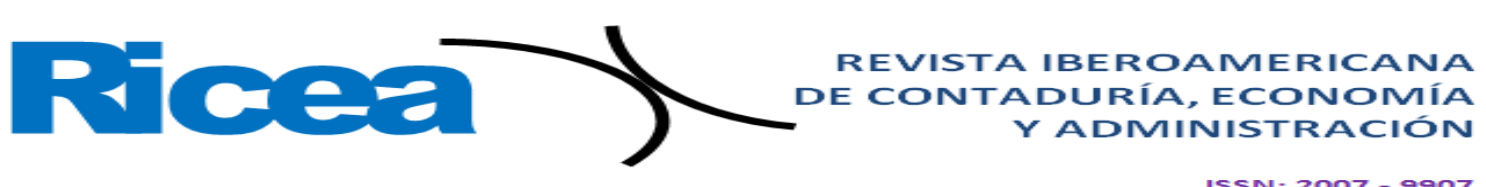

marginación y pobreza, los cuales se enfocan en conocer el bienestar de las personas según el acceso que tengan o no a indicadores como vivienda, automóvil, computadora, teléfono celular, Internet, etc. Al respecto, estos autores consideran que dichas variables son incompletas porque no incluyen la valoración que las personas tienen de sus vidas, pensamientos y sentimientos.

Millán y Castellanos (2018) enfatizan que los factores socioeconómicos no pueden ser medulares para la medición del bienestar, porque si ese fuera el parámetro sería factible suponer que una nación con un mayor producto interno bruto (PIB) tendría una población con mayores niveles de bienestar, lo cual no ha sido avalado en ponderaciones específicas. Precisan los citados investigadores que mediciones efectuadas en China hacia el año 2000 desmintieron esa teoría, pues se demostró que en esa nación algunos incrementos del PIB podían reducir los niveles de satisfacción reportados por su población. Por ello, subrayan que el incremento de ingresos no se traduce necesariamente en un aumento del bienestar, porque este implica satisfacción y condiciones cognitivas y emocionales, aspectos no valorados por el enfoque socioeconómico del bienestar.

En efecto, a las empresas les interesa principalmente atender el tema relacionado con el bienestar debido a que existen alarmantes estadísticas que asocian este problema con los costos ocasionados por enfermedades cada vez más recurrentes, como depresión, trastornos digestivos vinculados con el estrés y ausentismo laboral, lo cual les está generando grandes pérdidas económicas a las empresas, fenómeno que para las mypes puede ser catastrófico (Dagenais-Desmarais y Savoie, 2012).

Por su parte, Dagenais-Desmarais y Savoie (2012) ofrecen un modelo del bienestar psicológico en el trabajo basado en cómo se ha desarrollado el marco teórico de referencia sobre los enfoques que han prevalecido en la investigación y en la forma en que se han abordado para adaptarlos a los estudios organizacionales. Estos autores han propuesto los siguientes cinco factores para determinar el bienestar psicológico en el dominio laboral: ajuste interpersonal en el trabajo, prosperidad laboral, sentimiento de competencia, deseo de participación y percepción de reconocimiento en el trabajo. Para dicha propuesta los citados autores se han basado en las principales escalas validadas y aceptadas en otros trabajos, como el índice de bienestar psicológico usado en el trabajo de Crocker y Algina (1986), la escala de manifestación de bienestar psicológico de Massé et al. (1998a, 1998b), la escala psicológica de manifestación de angustia de Massé et al. (1998a, 1998c), el programa de

\section{(c) $\rightarrow \&$}




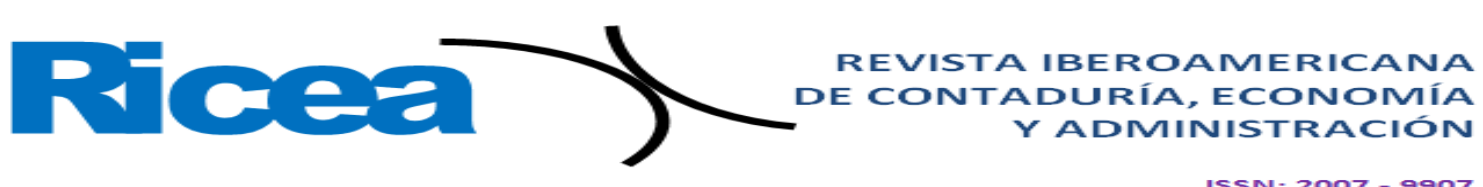

afectos positivos y negativos de Watson et al. (1988), así como la escala de satisfacción con la vida de Diener et al. (1985), todos referidos en Dagenais-Desmarais y Savoie (2012), lo que ha servido para conseguir un sustento sólido para la conceptualización del bienestar psicológico subjetivo en el trabajo.

Asimismo, pero en años más recientes —como mencionan Llorens, Salanova y Lasilla (2009) — ha crecido el interés por estudiar el rol del líder para crear ambientes de bienestar psicológico en el trabajo. De hecho, se ha analizado esa influencia para gestionar organizaciones que se caracterizan por invertir esfuerzos de colaboración, para maximizar el bienestar de los empleados y con ello impactar en la productividad, lo cual genera puestos bien diseñados y significativos, así como mejores climas organizacionales que evidencian las oportunidades equitativas y accesibles para desarrollar los propósitos personales como los de carrera o el balance vida-trabajo.

Para Perilla y Gómez (2017), el tipo de liderazgo a considerar para propiciar el bienestar psicológico en el trabajo es el transformacional, el cual fue propuesto por Burns en 1978 y posteriormente desarrollado por Bass en 1985, quien hizo una diferencia clara entre este estilo y el transaccional. Ambos autores consideran que el liderazgo transformacional consiste en generar una relación de participación y compromiso que convierte a los seguidores en líderes y puede convertir a los líderes en agentes morales.

Según Bass (1985), a través del liderazgo transformacional el líder motiva a los seguidores para obtener un desempeño que supera las expectativas al transformar las actitudes y los valores de los seguidores. Este estilo de influencia destaca un proceso a través del cual se promueve que los seguidores se distingan a ellos mismos como los retos y oportunidades de su entorno.

Por su parte, Lapo (2015) considera que el liderazgo transformacional se vincula con la capacidad de conducir a las personas de una organización para alcanzar los objetivos de esta. Para ello, la dirección deberá de estar basada en el respeto al líder, el cual surge por sus cualidades profesionales y personales, así como por sus conocimientos y capacidades para transmitir sus valores. Un liderazgo transformacional, en consecuencia, representará un proceso estratégico para asegurar el éxito de la empresa.

De la misma forma, el líder transformacional es capaz de ir más allá de los propios intereses inmediatos a través de su influencia y carisma, inspiración, estímulo intelectual o consideración individualizada, con lo cual logra elevar el nivel de madurez y de ideales del

\section{(C) $\rightarrow \Leftrightarrow \Leftrightarrow$}




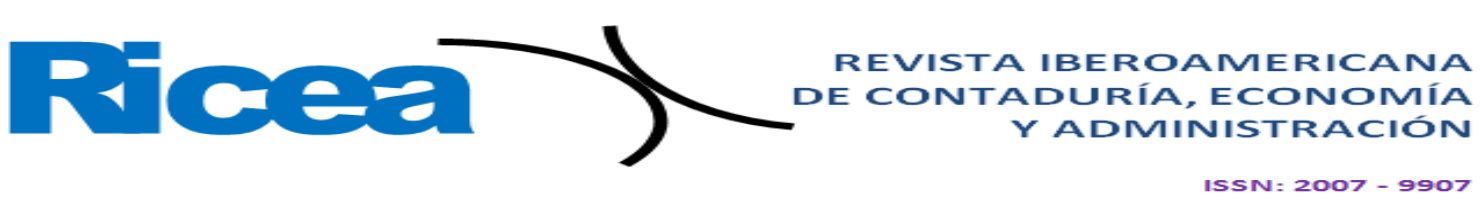

seguidor. Además, el líder transformacional está capacitado para transmitir su preocupación por el logro, la autorrealización y el bienestar de los demás, de la organización y de la sociedad, elementos esenciales para ganarse la credibilidad y el compromiso de sus seguidores.

Aun así, vale destacar que son escasos los estudios sobre el proceso a través del cual el liderazgo transformacional produce cambios en indicadores del bienestar psicológico en el trabajo. En este sentido, Sivanathan, Arnold, Turner y Barling (2004) han propuesto una serie de procesos psicológicos de los seguidores que son estimulados por el comportamiento de los líderes y que podrían mediar en la relación entre estas variables: la confianza en el líder, el trabajo significativo, la identidad organizacional y ocupacional, entre otras.

La confianza en el líder, específicamente, se ha asociado con el liderazgo transformacional, ya que parece ser clave para que esta forma de liderazgo sea efectiva. Podsakoff, Mackenzie, Moorman y Fetter (1990) destacan en su investigación que el compromiso que tenga el seguidor dependerá de la capacidad del líder para construir confianza entre ellos. Otras investigaciones además ponen de manifiesto la relación de la confianza con resultados organizacionales positivos (p. ej., el desempeño), así como con mejores condiciones psicológicas y de interrelación personal en el trabajo (Abbott y Freeth, 2008; Salanova, 2008).

Sin embargo, aún es escasa la evidencia empírica para determinar el papel del líder como mediador entre el liderazgo transformacional y el bienestar psicológico en el trabajo de los empleados (Braun, Peus, Weisweiler y Frey, 2013; Gilstrap y Collins, 2012). De hecho, Perilla y Gómez (2017) — citando a Kelloway, Turner, Barling y Loughlin (2012)— mencionan que su investigación es la única que explora la relación del liderazgo transformacional con el bienestar psicológico de los empleados, lo cual se concretó con un grupo de 436 trabajadores de una empresa de telecomunicaciones canadiense. No obstante, vale acotar que el bienestar psicológico se midió con el cuestionario general de salud (Goldberg, 1972), un instrumento diseñado para evaluar problemas de salud mental a través del reporte de síntomas de malestar, en lugar del bienestar psicológico propiamente dicho (García, 1999). Los resultados mostraron una mediación total de la confianza en la relación entre el liderazgo transformacional y el bienestar de los empleados (más exactamente, la ausencia de malestar).

\section{(C) $\rightarrow$ FO $\mathrm{NO}$}




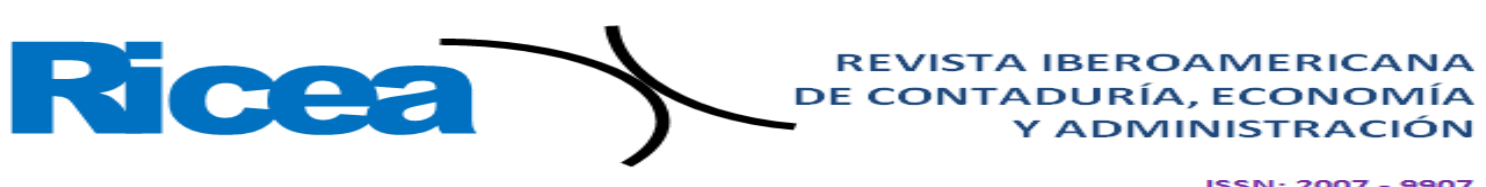

Por ese motivo, en la presente indagación se incluye la variable de la confianza en el líder como mediadora entre el estilo de liderazgo transformacional y el bienestar psicológico en el trabajo. En síntesis, este estudio ha sido diseñado con el propósito de aportar información —en un contexto de baja competitividad, como lo es el estado de Durangosobre la forma en que el bienestar psicológico en el trabajo supeditado a un liderazgo transformacional puede generar oportunidades para una gestión más efectiva como ventaja ante todas las limitaciones que deben afrontar las mypes.

\section{Metodología}

Se llevó a cabo un estudio cuantitativo, de corte transversal correlacional. En concreto, se realizó un estudio de caso, el cual se desarrolló mediante un censo aplicado a los trabajadores de una pequeña empresa industrial (ubicada el municipio de Durango, estado de Durango, México) que se encuentra operando desde 2011 en el mercado de la transformación de vidrio y aluminio. Cabe destacar que en sus inicios en la compañía solo operaban el actual gerente y el dueño empresario con un trabajador; sin embargo, a partir de 2015 pasaron de ser una microindustria a una pequeña industria, la cual en la actualidad se halla constituida por 25 empleados (únicamente dos trabajadores no fueron encuestados porque se encontraban fuera de la empresa: uno por permiso médico y otro por cuestiones laborales).

En cuanto a las características de la muestra, $91.3 \%$ estuvo compuesta por hombres y $8.7 \%$ por mujeres. La edad media de las personas fue de 33.4 años. En relación con el nivel educativo, $17.4 \%$ tenía estudios básicos (primaria y secundaria), $43.5 \%$ bachillerato/preparatoria general, $8.7 \%$ técnico superior universitario y $30.4 \%$ licenciatura. Asimismo, en sus actuales ocupaciones tienen un promedio de 30.1 meses. La recolección de los datos se realizó por medio de encuesta en un mismo momento para todas las variables y la fuente de información siempre fue la misma, es decir, los empleados.

\section{(c) 9 (5)}




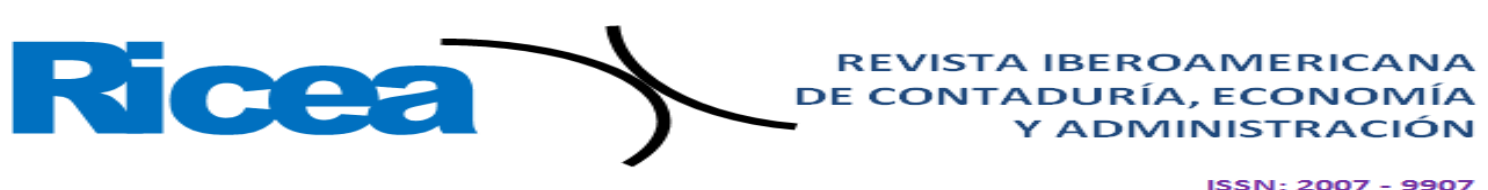

\section{Instrumentos de medición}

El cuestionario aplicado estuvo integrado por los siguientes componentes y escalas, se observan en la Tabla 1: la primera parte se usó para conocer las variables sociodemográficas (edad, sexo, nivel de estudios, tiempo laborando en la empresa, puesto ocupado en la actualidad). La segunda parte se empleó para determinar el liderazgo transformacional, para lo cual se utilizó la encuesta diseñada y validada por Podsakoff et al. (1990), que consta de 5 ítems medidos en una escala de tipo Likert del 1 al 5 con opciones de respuesta que van de nulo a totalmente. En la tercera parte se examinó la confianza en el líder utilizando la escala propuesta y validada por Cook y Wall (1980), específicamente la que mide la confianza en el gerente; esta se encuentra conformada por 6 ítems (dos de los cuales miden de manera inversa) con una escala Likert del 1 al 5 ( 1 es nulo y 5 totalmente). Por último, se adaptó la escala propuesta y validada por Dagenais-Desmarais y Savoie (2012) para medir el bienestar psicológico en el trabajo; esta escala está compuesta por 25 ítems clasificados en las siguientes 5 dimensiones: ajuste interpersonal en el trabajo, prosperidad laboral, sentimiento de competencia, reconocimiento percibido y deseo de participación en el trabajo, evaluados en una escala Likert del 1 al 5 (donde 1 es totalmente en desacuerdo y 5 totalmente de acuerdo).

Este instrumento fue empleado porque evalúa el bienestar psicológico únicamente en el ámbito laboral, es decir, separa los otros aspectos de la vida de una persona, con lo cual se puede evitar sesgar los resultados al evaluar positivamente ámbitos de la vida cotidiana en lugar del ámbito laboral.

\section{(C) $\rightarrow \& \Leftrightarrow$}




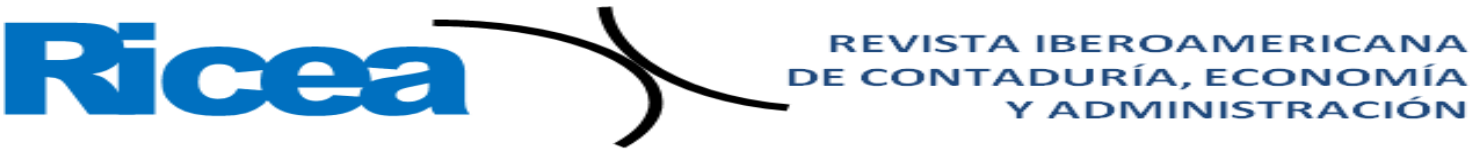

\section{Resultados}

Tabla 1. Cuadro de variables

\begin{tabular}{|c|c|c|c|}
\hline Variable & Dimensión & $\begin{array}{l}\text { N. }{ }^{\circ} \text { de preguntas en el } \\
\text { Instrumento }\end{array}$ & Tipo de variable \\
\hline $\begin{array}{l}\text { Perfil de los } \\
\text { participantes }\end{array}$ & $\begin{array}{l}\text { Variables } \\
\text { sociodemográficas }\end{array}$ & p 1 a la p 19 & Cualitativa/ordinal \\
\hline $\begin{array}{l}\text { Liderazgo } \\
\text { transformacional }\end{array}$ & 5 ítems & p20 a la p24 & Cualitativa/ordinal \\
\hline Confianza en el líder & 5 ítems & p25 a la p30 & Cualitativa/ordinal \\
\hline \multirow{5}{*}{$\begin{array}{l}\text { Bienestar psicológico en } \\
\text { el trabajo }\end{array}$} & Ajuste interpersonal & a18, a23, a28, a33, a38 & Cualitativa/ordinal \\
\hline & Prosperidad laboral & a19, a24, a29, a34, a39 & Cualitativa/ordinal \\
\hline & $\begin{array}{l}\text { Sentimiento de } \\
\text { competencia en el } \\
\text { trabajo }\end{array}$ & $\mathrm{a} 20, \mathrm{a} 25, \mathrm{a} 30, \mathrm{a} 35, \mathrm{a} 40$ & Cualitativa/ordinal \\
\hline & $\begin{array}{l}\text { Reconocimiento } \\
\text { percibido en el trabajo }\end{array}$ & a21, a26, a31, a36, a41 & Cualitativa/ordinal \\
\hline & $\begin{array}{l}\text { Deseo de participación } \\
\text { en el trabajo }\end{array}$ & $\mathrm{a} 22, \mathrm{a} 27, \mathrm{a} 32, \mathrm{a} 37, \mathrm{a} 42$ & Cualitativa/ordinal \\
\hline
\end{tabular}

Fuente: Elaboración propia

Se hicieron análisis descriptivos y se calcularon las medias de cada variable, se presentan en la Tabla 2; también se evaluaron las correlaciones bivariadas entre las variables de estudio.

Tabla 2. Estadísticos

\begin{tabular}{|l|r|r|r|}
\hline & \multicolumn{1}{|c|}{$\begin{array}{c}\text { Liderazgo } \\
\text { transformacional }\end{array}$} & $\begin{array}{c}\text { Confianza en el } \\
\text { líder }\end{array}$ & $\begin{array}{c}\text { Bienestar } \\
\text { psicológico en el } \\
\text { trabajo }\end{array}$ \\
\hline Media & 4.2174 & 3.3188 & 4.3409 \\
\hline Desviación estándar & .68201 & .39859 & .65968 \\
\hline
\end{tabular}

Fuente: Elaboración propia

Tabla 3. Correlaciones entre variables

\begin{tabular}{|l|r|r|}
\hline & $\begin{array}{c}\text { Liderazgo } \\
\text { transformacional }\end{array}$ & $\begin{array}{c}\text { Confianza en el } \\
\text { líder }\end{array}$ \\
\hline Liderazgo transformacional &, $653^{* *}$ & \\
\hline Confianza en el líder &, $660^{* *}$ &, $506^{*}$ \\
\hline Bienestar psicológico en el trabajo & \\
\hline **. La correlación es significativa en el nivel 0,01 (2 colas). \\
\hline *. La correlación es significativa en el nivel 0,05 (2 colas).
\end{tabular}

Fuente: Elaboración propia 


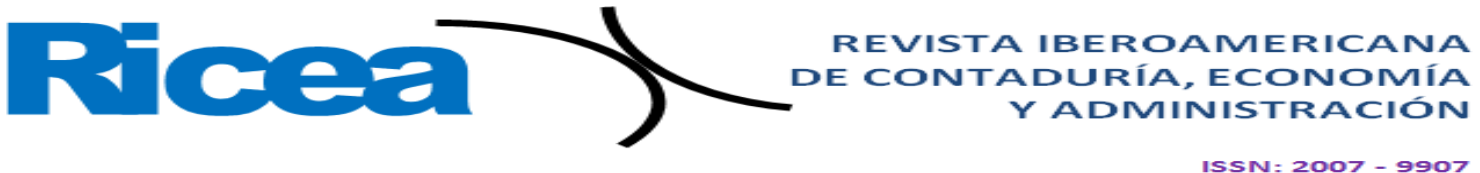

En la Tabla 3 se observa que el coeficiente de correlación de Pearson entre las variables liderazgo transformacional y bienestar psicológico en el trabajo es de ,660** Entre liderazgo transformacional y confianza en el líder es de, $653^{* *}$ y de confianza en el líder con bienestar psicológico en el trabajo de ,506*.

Como los valores de Pearson van de -1 a 1 - siendo 0 indicador de que no existe correlación-, se aprecia que entre estas dos variables existe una correlación significativa. La dirección de la correlación es positiva, es decir, directa. Por lo tanto, si aumenta la percepción de los colaboradores en una variable, aumenta en la otra (y viceversa).

Tabla 4. Correlaciones entre dimensiones de bienestar psicológico en el trabajo

\begin{tabular}{|l|c|c|c|c|}
\hline & $\begin{array}{c}\text { Ajuste } \\
\text { interpersonal }\end{array}$ & $\begin{array}{c}\text { Prosperidad } \\
\text { laboral }\end{array}$ & $\begin{array}{c}\text { Sentimiento } \\
\text { de } \\
\text { competencia } \\
\text { en el } \\
\text { trabajo }\end{array}$ & $\begin{array}{c}\text { Reconocimiento } \\
\text { percibido en el } \\
\text { trabajo }\end{array}$ \\
\hline Ajuste interpersonal &, $943^{* *}$ & & & \\
\hline Prosperidad laboral &, $895^{* *}$ &, $909^{* *}$ & & \\
\hline $\begin{array}{l}\text { Sentimiento de competencia en el } \\
\text { trabajo }\end{array}$ &, $748^{* *}$ &, $739^{* *}$ &, $813^{* *}$ &, $895^{* *}$ \\
\hline Reconocimiento percibido en el trabajo &, $911^{* *}$ &, $886^{* *}$ & & \\
\hline Deseo de participación en el trabajo & & & \\
\hline **. La correlación es significativa en el nivel $0,01(2$ colas).
\end{tabular}

Fuente: Elaboración propia

Para interpretar los datos de la Tabla 4, se observa que el coeficiente de correlación de Pearson entre las dimensiones de la variable bienestar psicológico en el trabajo es una correlación alta, ya que el valor es cercano a 1.

\section{Discusión}

Se le aplicó a la encuesta el coeficiente de alfa de Cronbach, el cual ha sido históricamente utilizado para evaluar las escalas de medida. Valores elevados de este coeficiente indican, en principio, una alta fiabilidad de la escala de medida, aunque vale destacar que este por sí solo no refiere la validez de las escalas utilizadas. Por ello, cada variable está acreditada por la validez que han puesto a prueba los autores de quienes se tomaron los instrumentos, los cuales han realizado estudios que sirvieron de sustento para investigaciones posteriores. De igual manera, las correlaciones presentadas se realizan para

\section{(c) $\rightarrow \Leftrightarrow \Leftrightarrow$}




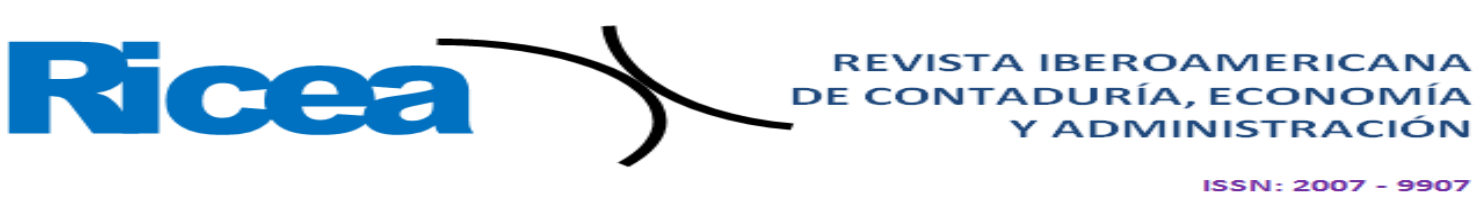

enfatizar si es verdad que en las hipótesis planteadas existe un rango de compatibilidad para promover futuros estudios bajo el mismo tenor que se plantea en la investigación.

Ahora bien, como ya se mencionó, en la primera parte del instrumento se analizaron las variables sociodemográficas, lo cual arrojó unos datos que coincidieron con lo señalado por el Inegi (2018), institución que indica que la industria en México está compuesta en su mayoría por hombres. En la presente indagación, además, se cumplió la observación del Plan Estatal de Desarrollo para Durango 2016-2022, donde se señala que en esta entidad federativa las industrias están compuestas principalmente por personal con estudios básicos, bachillerato o técnicos. Es decir, los trabajadores no suelen haber cursado la educación superior o la especialización profesional como determinante para la capacitación y el desarrollo. Aunado a ello, la edad promedio es de 33 años, lo que indica que el personal no se encuentra en el promedio de edad para cursar los estudios prevalecientes en la población de la industria analizada.

Por otra parte, con los resultados de la segunda de este trabajo — referente al liderazgo transformacional—, se puede afirmar que el líder motiva y toma en cuenta a sus trabajadores para realizar sus funciones, tal como lo han apuntado Podsakoff et al. (1990), así como Avolio y Bass (2002). En otras palabras, se observa que el liderazgo transformacional tiene una correlación aceptable y significativa con la confianza en el líder. Igualmente, existe una correlación aceptable entre el liderazgo transformacional y el bienestar psicológico en el trabajo, lo cual es positivo, ya que — como mencionan Perilla y Gómez (2017) — el liderazgo transformacional puede afectar especialmente en el bienestar afectivo de los empleados y en la prevención de trastornos físicos asociados a la salud.

Asimismo, cabe destacar que el hecho de que aparezcan las tres variables enlazadas (es decir, una correlación aceptable y significativa entre el liderazgo transformacional y la confianza con el líder, así como una correlación aceptable y significativa entre el liderazgo transformacional y el bienestar psicológico en el trabajo) invita a desarrollar más estudios en este tipo de empresas, pues de esa manera se puede concluir si esta es una fortaleza para la supervivencia y el crecimiento del negocio.

Anteriormente, se mencionó que la pequeña industria objeto de esta investigación pasó (en un periodo de 7 años) de 2 a 26 trabajadores, es decir, de microempresa a pequeña empresa, lo cual demuestra un notable crecimiento en cuanto a su economía y capital humano.

\section{(c) $\rightarrow$ Fo}




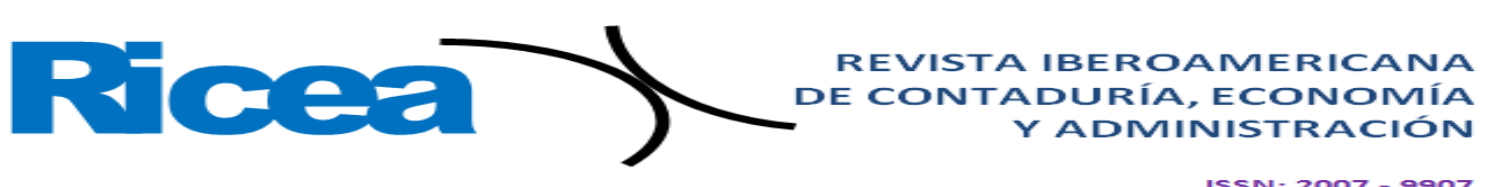

Por último, en lo concerniente a la variable del bienestar psicológico en el trabajo, se puede notar una evaluación positiva general, lo que refleja un buen ambiente laboral. En este sentido, se puede afirmar que los empleados - aunque en su mayoría operativos - tienen un sentimiento positivo en su ámbito laboral. De hecho, al realizar las correlaciones entre las dimensiones propias de la variable, se observó que los trabajadores mantienen un elevado deseo de participación en las labores, así como un sentimiento de competencia y prosperidad laboral, aspectos que buscan satisfacer por encima de las otras dimensiones no menos valoradas.

\section{Conclusiones}

Además de las disquisiciones teóricas de la investigación propuesta que destacan la relevancia del proceso de liderazgo en el bienestar psicológico de los trabajadores, también se puede ofrecer una reflexión para las implicaciones prácticas, como la necesidad de formación, capacitación y aprendizaje del líder en el marco de un estilo transformacional, pues de ese modo podrá no solo saber comunicarse de forma inspiradora, sino también estimular intelectualmente a sus trabajadores, así como apoyarlos y reconocer la labor que realizan para conseguir los objetivos organizacionales.

En concordancia con esta observación, se puede asegurar que aquellas empresas que cuenten con líderes transformacionales tendrán mayor cuidado del capital psicológico positivo y podrán ser categorizadas como organizaciones sanas. Esto se puede inferir de los resultados conseguidos en este estudio, los cuales sirven para apoyar la comprobación de las hipótesis.

Además, cuando los trabajadores sienten esa percepción de cuidado, podrán generar mayores niveles de afecto positivo y compromiso, lo cual posiblemente se suele traducir en más elevados estándares de desempeño. Esta, sin embargo, es una la línea de investigación que queda planteada para próximos trabajos.

Otro estudio que se debe desarrollar en el futuro tiene que ver con el análisis del desempeño organizacional y las percepciones del gerente respecto a la participación y desarrollo de los trabajadores. Esto significa que se debe poner a prueba la relación que existe entre el liderazgo transformacional, el bienestar psicológico en el trabajo y el desempeño organizativo.

\section{(c) $\rightarrow \Leftrightarrow$}




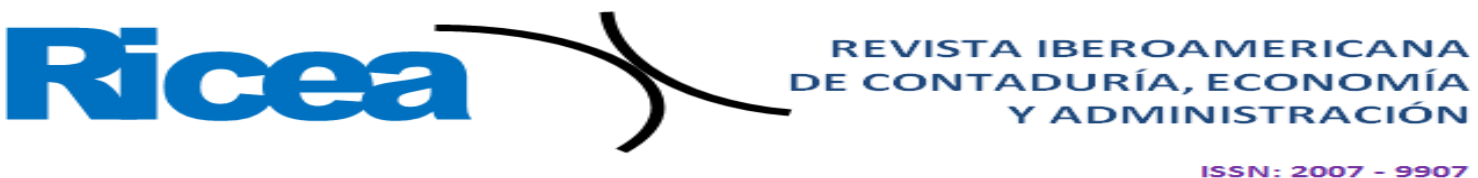

Esta investigación de caso, además de probar las hipótesis propuestas en la introducción, constituye un precedente para el estudio organizacional a partir de variables de orden subjetivo, las cuales pueden constituir una fortaleza y una ventaja ante la necesidad de las mypes de utilizar herramientas que no sean costosas para el negocio. Este es un capital activo que se debe tomar en cuenta para participar y proveer a la organización de herramientas que en ocasiones el líder o gerente no puede aportar por sí mismo, las cuales pueden ser gestionadas desde la visión de un liderazgo transformacional.

Asimismo, se debe tener presente que fomentar entre el personal altos niveles de bienestar psicológico puede servir como medida preventiva de múltiples enfermedades de tipo mental o asociadas el estrés laboral, las cuales resultan muy costosas para ser cubiertas por las mypes. Todas estas iniciativas, en definitiva, proveen recursos potenciales para diagnosticar del bienestar psicológico en el trabajo, con un rigor metodológico que se apoya en la métrica no solo para comprender las nociones de ese tema, sino también para plantear estrategias organizacionales en las mypes.

\section{(c) 9 (5)}




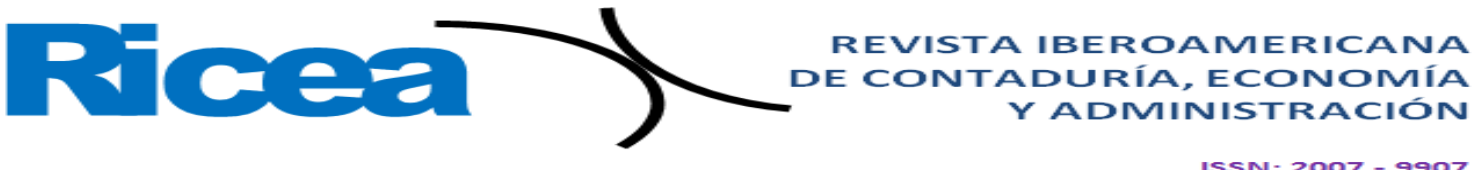

\section{Referencias}

Abbott, S. and Freeth, D. (2008). Social Capital and Health. Journal of Health Psychology, 13(7), 874-883. Doi: https://doi.org/10.1177/1359105308095060

Adler, A., Unanue, W., Osin, E., Ricard, M., Alkire, S. and Seligman, M. (2013). Psychological well-being. In Royal Government of Bhutan (ed.), Psychological wellbeing. In Report on Wellbeing \&amp; Happiness (pp. 101-136). Retrieved from http://www.tapartsproject.co.uk/data/dynamic/spaw/documents/UN\%20Wellbeing \%20Report.pdf.

Adler, M., Dolan, P. and Kavetsos, G. (2014). Understanding life choices: Happiness or something else? London School of Economics: Mimeo.

Avendaño, B. L. y Perrotini, I. (2015). Insuficiencia dinámica, crecimiento y desempleo en México, 1974-2012. Investigación Económica, 74(293), 99-130. Recuperado de www.redalyc.org/articulo.oa?id=60141352006

Avolio, B. and Bass, B. (eds.) (2002). Developing potential across a full range of leadership: cases on transactional and transformational leadership. Mahwah, N. J.: Lawrence Erlbaum Associates.

Barley, S. y Kunda, G. (1992). Estructura y diseño vs. lealtad y sentimiento: oleadas de ideologías racionales y normativas de control en el discurso gerencial. Tecnología Administrativa, 9(29), 135-192.

Bass, B. M. (1985). Leadership and performance beyond expectations. New York, New York: Free Press.

Braun, S., Peus, C., Weisweiler, S. and Frey, D. (2013). Transformational leadership, job satisfaction, and team performance: A multilevel mediation model of trust. The Leadership Quarterly, 24(1), 270-283. Doi: https://doi.org/10.1016/j.leaqua.2012.11.006

Castellanos, R. y Rojas, M. (2018). Bienestar subjetivo como perspectiva del progreso social. En Millán, R. y Castellanos, R. (eds.), Bienestar subjetivo en México (pp. 21-53). Ciudad de México, México: Instituto de Investigaciones Sociales.

\section{(C) $\rightarrow \& \Leftrightarrow$}




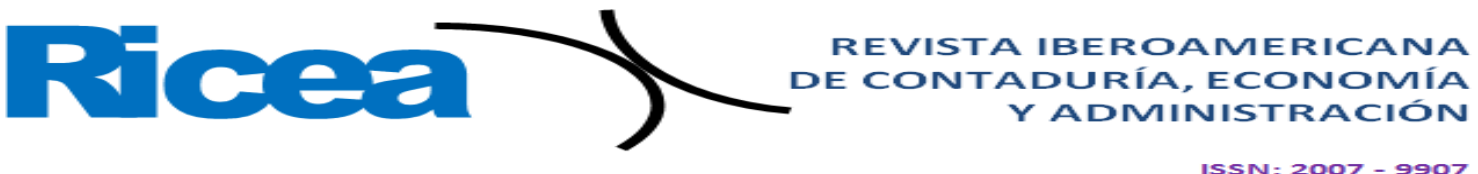

Castilla, A. (2014). Optimización del talento en las organizaciones: una mirada desde la realidad de la dirección de RR. HH. Athenea Digital, 14(2), 285-291.

Comisión Económica Para América Latina y el Caribe [Cepal] (2018). Perspectivas económicas de América Latina 2018 Repensando las instituciones para el desarrollo. París: Éditions OCDE.

Cook, J. and Wall, T. (1980). New work attitude measures of trust, organizational commitment and personal need non-fulfilment. Journal of Occupational Psychology, 53(1), 39-52. Doi: https://doi.org/10.1111/j.2044-8325.1980.tb00005.x

Dagenais-Desmarais, V. and Savoie, A. (2011). What is Psychological Well-Being, Really? A Grassroots Approach from the Organizational Sciences. Journal of Happiness Studies, 13(4), 659-684. https://doi.org/10.1007/s10902-011-9285-3

Diener, E. and Biswas-Diener, R. (2002). Will money increase subjective well-being? A literature review and guide to needed research. Social Indicators Research, 57(2), 119-169. Doi: https://doi.org/10.1023/a:1014411319119

Diener, E. and Seligman, M. (2004). Beyond money: toward an economy of well-being. Psychological Science in the Public Interest, 5(1), 1-25.

García, C. (1999). Manual para la utilización del cuestionario de salud general de Goldberg. Adaptación cubana. Revista Cubana de Medicina General Integral, 15(1), 88-97.

Gilstrap, J. B., y Collins, B. J. (2012). The Importance of Being Trustworthy. Journal of Leadership \& Organizational Studies, 19(2), 152-163. Doi: https://doi.org/10.1177/1548051811431827

Gobierno del Estado de Durango (2016). Plan Estatal de Desarrollo, 2016-2022 (ed. rev.). Durango, México: Gobierno del Estado de Durango.

Goldberg, D. P. (1972). The detection of psychiatric illness by questionnaire: A technique for the identification and assessment of non-psychotic psychiatric illness (rev. ed.). Oxford, England: Oxford University Press.

Instituto Nacional de Geografía y Estadística [Inegi] (2018). Encuesta nacional sobre productividad y competitividad de las micro, pequeñas y medianas empresas (ENAPROCE).

Recuperado

de

\section{(c) $9 \Leftrightarrow \theta$}




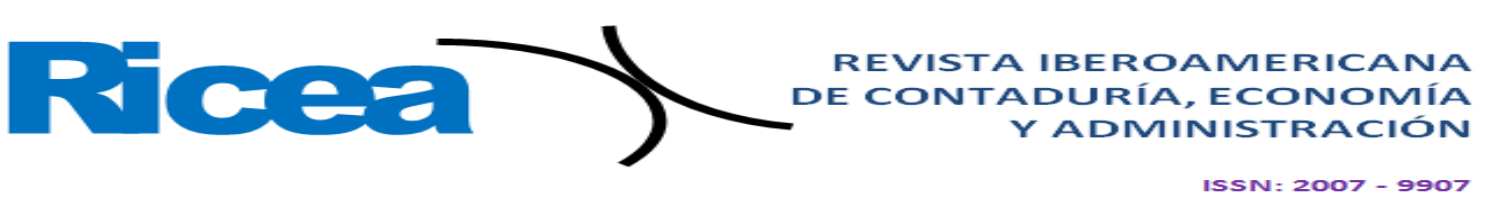

http://www.inegi.org.mx/est/contenidos/proyectos/encuestas/establecimientos/otras/ enaproce/

Lapo, M. (2015). El liderazgo y su evolución histórica. Revista Empresarial, 9(36), 11-16.

Llorens, S., Salanova, M. y Lasilla, J. (2009). Liderazgo transformacional y capital psicológico positivo: un estudio de caso en una empresa de construcción. Revista Directivos Construcción, (220), 48-58.

Millán, R. y Castellanos, R. (2018). Bienestar subjetivo en México. Ciudad de México, México: Instituto de Investigaciones Sociales.

Moreno, Z. e Ibarra, L. E. (2016). Fiabilidad y validez de la escala de felicidad de Lima en trabajadores de empresas de servicios. Desarrollo Gerencial, 9(1), 16-34.

Organización Internacional del Trabajo [OIT] (2018). Perspectivas sociales y del empleo en el mundo: $\quad 2018 . \quad$ Rendencias de http://www.ilo.org/global/research/global-reports/weso/trends-forwomen2018/WCMS_619603/lang--es/index.htm

Organización para la Cooperación y el Desarrollo Económico [OCDE] (2018). Getting it Right: Prioridades estratégicas para México. Éditions OCDE, Doi: https://doi.org/10.1787/9789264292871-es

Peiró, J. M., Tordera, N., Lorente, L., Rodríguez, I. y Ayala, Y. (2014). Bienestar sostenible en el trabajo: revisión y reformulación. Papeles del Psicólogo, 35(1), 5-14. Retrieved from http://www.redalyc.org/articulo.oa?id=77830184002

Perilla, L. E. y Gómez, V. (2017). Relación del estilo de liderazgo transformacional con la salud y el bienestar del empleado: el rol mediador de la confianza en el líder. Revista de Psicología del Trabajo y de las Organizaciones, 33(2), 95-108. Doi: https://doi.org/10.1016/j.rpto.2017.02.005

Podsakoff, P. M., MacKenzie, S. B., Moorman, R. H. and Fetter, R. (1990). Transformational leader behaviors and their effects on followers' trust in leader, satisfaction, and organizational citizenship behaviors. The Leadership Quarterly, 1(2), 107-142. https://doi.org/10.1016/1048-9843(90)90009-7

\section{(c) क्Sल)}




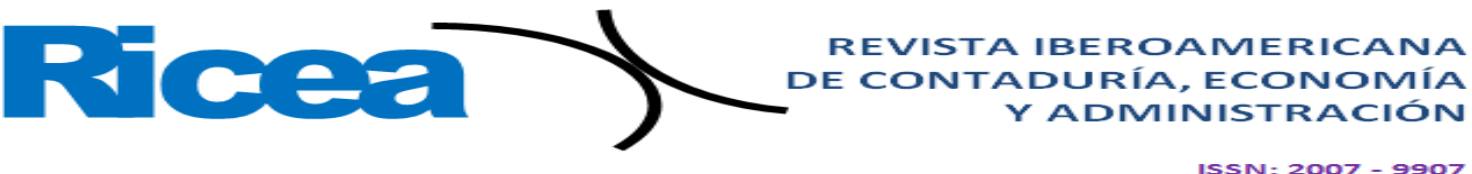

Posada, R., Aguilar, O. C. y Peña, N. B. (2016). Análisis sistémico de la micro y pequeña empresa en México. México, México: Pearson.

Salanova, M. (2008). Organizaciones saludables: una perspectiva desde la psicología positiva. En Vázquez, C. y Hervás, G. (eds.), Psicología positiva aplicada (rev. ed., pp. 1-15). Bilbao, Bilbao: Desclée de Brouwer.

Sansores, E. A. y Navarrete, J. E. (2018). Crecimiento de las micro, pequeñas y mediana empresas: un análisis de los factores determinantes. Revista Venezolana de Gerencia, 23(81), 1-33. Recuperado de http://www.redalyc.org/jatsRepo/290/29055767010/index.html

Seligman, M., Ernst, R., Gillham, J., Reivich, K. and Linkins, M. (2009). Positive education: Positive psychology and classroom interventions. Oxford Review of Education, 35(3), 293-311.

Sivanathan, N., Arnold, K., Turner, N. and Barling, J. (2004). Leading Well: Transformational Leadership and Well-Being. In Linley, P. A. and Joseph, S. (eds.), Positive psychology in practice (pp. 241-255). Hoboken, NJ, US: John Wiley \& Sons Inc.

\section{(C) $\rightarrow \& \Theta$}




\begin{tabular}{|c|c|}
\hline Rol de Contribución & Definición. María Brenda González Herrera \\
\hline Conceptualización & $\begin{array}{l}\text { (principal) María Brenda González Herrera, (apoyo) José } \\
\text { Gerardo Ignacio Gómez Romero. }\end{array}$ \\
\hline Metodología & $\begin{array}{l}\text { (principal) María Brenda González Herrera, (apoyo) Ernesto } \\
\text { Geovani Figueroa González. }\end{array}$ \\
\hline Software & María Brenda González Herrera. \\
\hline Validación & $\begin{array}{l}\text { (principal) María Brenda González Herrera, (apoyo)Ernesto } \\
\text { Geovani Figueroa González. }\end{array}$ \\
\hline Análisis Formal & $\begin{array}{l}\text { (principal) María Brenda González Herrera, (apoyo)José } \\
\text { Gerardo Ignacio Gómez Romero, (apoyo)Ernesto Geovani } \\
\text { Figueroa González. }\end{array}$ \\
\hline Investigación & María Brenda González Herrera \\
\hline Recursos & María Brenda González Herrera. \\
\hline Curación de datos & María Brenda González Herrera. \\
\hline $\begin{array}{l}\text { Escritura - Preparación del } \\
\text { borrador original }\end{array}$ & María Brenda González Herrera. \\
\hline $\begin{array}{l}\text { Escritura - Revisión y } \\
\text { edición }\end{array}$ & $\begin{array}{l}\text { (principal) María Brenda González Herrera, (igual)José } \\
\text { Gerardo Ignacio Gómez Romero, (apoyo) Ernesto Geovani } \\
\text { Figueroa González. }\end{array}$ \\
\hline Visualización & María Brenda González Herrera. \\
\hline Supervisión & María Brenda González Herrera. \\
\hline $\begin{array}{l}\text { Administración de } \\
\text { Proyectos }\end{array}$ & María Brenda González Herrera. \\
\hline Adquisición de fondos & $\begin{array}{l}\text { (principal) María Brenda González Herrera, (apoyo) Ernesto } \\
\text { Geovani Figueroa González. }\end{array}$ \\
\hline
\end{tabular}

